\title{
ЗАДАЧА КЛАССИФИКАЦИИ ПОЛИМАТРОИДОВ
}

\author{
(Представил А. Хумал)
}

\section{§1. Постановка задачи}

Возросший интерес к исследованию комбинаторной структуры многогранников объясняется возможностью применения результатов для оценки трудоемкости методов оптимизации и для построения более эффективных алгоритмов решения задач оптимизации $\left[{ }^{1-8}\right]$. Мы развиваем идеи работ $\left[{ }^{9}\right]$ и $\left[{ }^{10}\right]$ исходя из определения полиматроида, приведенного в $\left[{ }^{11}\right]$ (оно же является основным в $\left[{ }^{1}\right]$, см. с. 142). Обозначим всюду через $\mathfrak{J}$ непустое конечное множество $\mathfrak{J}=\{1, \ldots, n\}$ и рассмотрим $n$-мерное действительное векторное пространство $R_{n}=$ $=\left\{\boldsymbol{x}=\left(x_{1}, \ldots, x_{n}\right), x_{i} \in R, i \in J\right\}$, где $R-$ множество всех действительных чисел. Модуль произвольного элемента $\boldsymbol{x} \in R_{n}$ определяется формулой

$$
|x|=\sum_{i \in J}\left|x_{i}\right|
$$

а частичный порядок вводится в $R_{n}$ следующим образом. Если для $\boldsymbol{x}, a \in R_{n}$ при всех $i \in \mathfrak{J}$ справедливо $x_{i} \leqslant a_{i}$, то вектор $\boldsymbol{x}$ называется подвектором вектора $\boldsymbol{a}$ и обозначается через $\boldsymbol{x} \leqslant \boldsymbol{a}$. Если не имеет места ни $\boldsymbol{x} \leqslant \boldsymbol{a}$, ни $\boldsymbol{a} \leqslant \boldsymbol{x}$, то векторы $\boldsymbol{x}, \boldsymbol{a} \in R_{n}$ называются несравнимыми и обозначаются через $\boldsymbol{x} \| \boldsymbol{a}$.

Пусть П - некоторое подмножество в $R_{n}$. Если элемент ${ }^{*} \boldsymbol{x} \in \Pi$ таков, что при любом $\boldsymbol{x} \in$ П либо $\boldsymbol{x} \leqslant{ }^{*} \boldsymbol{x}$, либо $\boldsymbol{x} \|^{*} \boldsymbol{x}$, то элемент ${ }^{*} \boldsymbol{x}$ называется максимальным в П.

O п р еде лен и е 1. Пусть Ј и $R$ означают те же множества, что и раньше. Пусть $\Pi$ - непустое компактное подмножество ${ }^{\circ} R^{+}{ }_{n}=$ $=\left\{x=\left(x_{1}, \ldots, x_{n}\right), \quad x_{i} \in R, \quad x_{i} \geqslant 0, \quad i \in J\right\} \subset R_{n}$. Пара $P=\{J, \Pi\}$ называется полиматроидом $c$ множеством независимых векторов П, если

1) любой подвектор независимого вектора является независимым,

2) все максимальные независимые подвекторы любого $а \in R^{+}{ }_{n}$ имеют одинаковый модуль.

Имеет место

Т ео рем а 1 (см. [11], с. 173). Множество П независимых векторов полиматроида $P$ является выпиклым многогранником в $R^{+}{ }_{n}$.

Этот многогранник П называется многогранником независимости полиматроида $P$. Если П не содержится ни в каком $(n-1)$-мерном подпространстве $R^{+}{ }_{n-1} \subset R^{+}{ }_{n}$, то полиматронд $P$ называется $n$-мерным. Обозначим множество всех $n$-мерных полиматроидов через $T(n)$. Поскольку по определению 1 любой полиматроид $P \in T(n)$ полностью определяется своим многогранником независимости $\Pi \subset R^{+}{ }_{n}$, то для полного описания полиматроида $P \in \mathfrak{T}(n)$ достаточно описать его вы- 
пуклый многогранник независимости П. Поэтому в дальнейшем мы сосредоточим свое внимание на исследовании многогранников независимости $\Pi \subset R^{+}{ }_{n}$ полиматроидов $P \in \mathfrak{T}(n)$. Мы будем их называть просто n-мерными полиматроидами.

Оказывается, что $n$-мерный полиматроид П как выпуклый многогранник пространства $R^{+}{ }_{n}$ задается с помощью субмодулярной функшии.

О пре деление 2. Если через 2(ग) обозначено мнонество всех подмножеств множества э и задано отображение $r: 2(\mathfrak{J}) \rightarrow R$, удовлетворяющее условиям $r(\varnothing)=0, r(x) \leqslant r(y)$ при всех $x \subseteq \mathrm{Y} \in 2(\mathfrak{I})$, то это отображение называется нецбывающей субмодулярной функцией над п-элементным множеством $J$, если при любых $\mathfrak{x}, \mathrm{y} \in 2(\mathfrak{J})$ имеет место

$$
r(x \cup y)+r(x \cap y) \leqslant r(x)+r(y) \text {. }
$$

Те о рем а 2 (см. $\left[{ }^{1}\right]$, с. 144$)$. Многогранник $\Pi \subset R^{+}{ }_{n}$ является $n$-мерным полиматроидом тогда и только тогда, когда существует такая неубывающая субмодулярная функиия $r: 2(\mathfrak{J}) \rightarrow R$, что

$$
\Pi=\left\{x \in R^{+} n, \sum_{i \in \mathfrak{X}} x_{i} \leqslant r(X) \text { при всех } X \in 2(\mathfrak{J})\right\} .
$$

Таким образом, будем говорить, что любой полиматроид задается системой

$$
\sum_{i \in X} x_{i} \leqslant r(x), \quad x \in 2(J)
$$

Рассмотрим множество всех перестановок из элементов множества $J$ и обозначим его через

$$
\mathrm{S}_{n}=\{\sigma=(1 \sigma, \ldots, n \sigma), k \sigma \in J, k \in J\} .
$$

Определим с помощью произвольно фиксированной перестановки $r \in S$ спенияльные подмножества множестра $J$ А именно, пусть

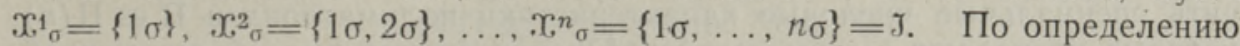
$\mathfrak{X}^{i}{ }_{\sigma} \in 2(\jmath)$ при любом $i \in \mathfrak{J}$. Если дополнительно считать, что $\mathfrak{X}^{0}{ }_{\sigma}=\varnothing$, то $\left\{x_{\sigma}^{i}, i=0, \ldots, n ; \sigma \in \mathrm{S}_{n}\right\}=2(\jmath)$. Справедлива следующая

Теорем а 3 (см. $\left[{ }^{1}\right]$, с. 145 ). Пусть мнпгогранник $\Pi \subset R^{+}{ }_{n}$ задан. системой (2). Вектор $\boldsymbol{x}=\left(x_{1}, \ldots, x_{n}\right) \in R^{+}{ }_{n}$ является его вершиной тогда и только тогда, когда существует перестановка $\sigma \in \mathrm{S}_{n}$ и иелое число $0 \leqslant k \leqslant n$ такие, "то компоненты $x_{s \sigma}(s \sigma \in J)$ вектора $\boldsymbol{x}$ вычисляются по формулам

$$
\begin{aligned}
& x_{s \sigma}=r\left(x_{\sigma}^{s}\right)-r\left(x^{s-1}\right) ; \quad s=1, \ldots, k, \\
& x_{s \sigma}=0, \quad s=k+1, \ldots, n,
\end{aligned}
$$

где $r(x) \quad(x \in 2(\mathfrak{x}))$ является неубывающей субмодулярной функцией из системы (2).

Задача классификации $n$-мерных полиматроидов равносильна задаче классификации тех выпуклых многогранников $\Pi \subset R^{+}{ }_{n}$, которые задаются системой (3). Обозначим множество всех таких многогранников через $\Pi(n)$. Тогда задача классификации является фактически задачей разбиения множества $\Pi(n)$ на такие подмножества, которые состояли бы из полиматроидов, являющихся в определенном смысле одинаковыми. Естественным путем получения классификашии окажется задание на множестве П $(n)$ некоторого отношения эквивалентности, т. е. бинар- 
ного отношения, являющегося рефлексивным, симметричным и транзитивным. Тогда возникнут классы эквивалентности как множества полиматрондов, эквивалентных между собой в рассматриваемом смысле. Эти классы считаю́тся классами соответствующей классификации. Целесообразно, чтобы полученная классификация полиматроидов удовлетворяла некоторым дополнительным условиям, а именно, следует требовать

1) конечность классификации, означающую конечность числа классов эквивалентности;

2) приводимость классификации, означающую инвариантность классов эквивалентности относительно действия группы перестановок (перенумеращий) координатных осей в пространстве $R_{n}$;

3) гомотетичность классификации, означающую инвариантность классов эквивалентности относительно действия группы гомотетий пространства $R_{n}$ с положительными параметрами (группы положительных гомотетий).

О п ределение 3 . Говорят, что полиматроид $\Pi^{\prime} \in \Pi(n)$ получается из полиматроида $\Pi \in \Pi(n)$ перестановкой $\omega \in S_{n}$ координатных осей приводимой и гомотетичной тогда и только тогда, когда существуют $\boldsymbol{x}^{\prime}=\left(x_{1}^{\prime}, \ldots, x_{n}^{\prime}\right)=\left(x_{1 \omega}, \ldots, x_{n \omega}\right) \in \Pi^{\prime} \quad\left(\right.$ обозначение $\left.\Pi^{\prime}=\Pi \omega\right)$. Говорят, что полиматроид $\Pi^{\prime} \in \Pi(n)$ получается из полиматроида $\Pi \in \Pi(n)$ положительной гомотетией пространства $R_{n}$, если существует такое число $\mathrm{\varrho} \in(0,+\infty)$, что $\boldsymbol{x}=\left(x_{1}, \ldots, x_{n}\right) \in \Pi$ тогда и только тогда, когда $\boldsymbol{x}^{\prime}=\left(x_{1}^{\prime}, \ldots, x_{n}^{\prime}\right)=\left(\varrho x_{1}, \ldots, \varrho x_{n}\right) \in \Pi^{\prime}$ (обозначение $\left.\Pi^{\prime} \iota=\mathrm{QI}\right)$.

С учетом транзитивности отношения эквивалентности теперь легко доказывается следующее

Предложен и е 1. Классификация полиматроидов П $\in \Pi(n)$ является приводимой $и$ гомотетичной тогда и только тогда, когда существуют $\omega \in S_{n}$ и число $\varrho \in(0,+\infty)$ так, что если $\Pi^{\prime}=\varrho(\Pi \omega)=(\varrho \Pi) \omega$, то П и П' эквивалентны (обозначение $\left.\Pi \sim \Pi^{\prime}\right)$.

Для выяснения возможных общих принципов классификации на множестве П $(n)$ введем некоторые вспомогательные понятия, чтобы получить специальные числовые характеристики полиматроидов $\Pi \in \Pi(n)$, которые позволяют с геометрической точки зрения дать более подробное описание полиматрондов $\Pi \in \Pi(n)$, чем задание их системой $(2)$.

\section{§ 2. Многогранник максимальных элементов полиматроида}

Исходя из определения 1 , можно убедиться, что любой $n$-мерный полиматронд $\Pi \in \Pi(n)$ содержит максимальные элементы. Обозначим непустое множество этих элементов через *П. Множество *П описывается с помощью некоторых новых понятий. А именно, сопоставим произвольной перестановке $\sigma \in S_{n}$ элемент ${ }^{*} \boldsymbol{x}^{\sigma}$ рассматриваемого полиматроида $\Pi \in \Pi(n)$ так, чтобы его координаты $x^{\sigma}{ }_{k \sigma}(k \sigma \in J)$ вычислялись по формулам

$$
{ }^{*} x^{\sigma}{ }_{k \sigma}=\max _{x \in \Pi_{(1 \ldots(k-1)) \sigma}} x_{k \sigma}, \quad k \in J
$$

где по определению считаем

$$
\Pi_{0 \sigma} \equiv \Pi, \quad \Pi_{(1 \ldots(k-1)) \sigma}=\left\{x \in \Pi, x_{1 \sigma}={ }^{*} x^{\sigma}{ }_{1 \sigma}, \ldots, x_{(k-1) \sigma}={ }^{*} x^{\sigma}{ }_{(k-1) \sigma}\right\} .
$$

Основываясь на определении максимального элемента полиматронда $\Pi \subset R^{+}{ }_{n}$, можно показать, что ${ }^{*} \boldsymbol{x}^{\sigma}=\left({ }^{*} x^{\sigma}{ }_{1}, \ldots,{ }^{*} x^{\sigma}{ }_{n}\right) \in \Pi$ с координатами, вычисленными по (4), является максимальным элементом в П, и 
тем самым * $\boldsymbol{x} \in{ }^{*} \Pi$. Вектор $\boldsymbol{x}^{\sigma} \in \Pi$ называется $\sigma$-максимальным в $\Pi$. С помощью $\sigma$-максимальных элементов $\left\{{ }^{*} \boldsymbol{x}^{\sigma}, \sigma \in \mathcal{S}_{n}\right\}$ задается отношение эквивалентности на множестве $\boldsymbol{S}_{n}$.

О п ределени е 4. Перестановки $\sigma, \tau \in \mathrm{S}_{n}$ называются эквивалентными относительно полиматроида $\Pi \in \Pi(n)$ тогда и только тогда, когда

$$
{ }^{*} x_{i}^{\sigma}={ }^{*} x_{i}^{\tau} \text { nри всех } i=k \sigma=l \tau \in J, k, l \in J
$$

(эквивалентность обозначается через $\sigma \approx \tau$ ).

Пусть множество $S_{n}$ разбивается соответственно определенному отношению эквивалентности на $m$ классов эквивалентности. Очевидно, что $1 \leqslant m \leqslant n !=\left|S_{n}\right|$. Пусть $\mathscr{M}=\{1, \ldots, m\}$. Пронумеруем получаемые классы эквивалентности с помощью индексов $\alpha \in \mathcal{M}$ и обозначим через $E^{\alpha}(\Pi) \subseteq \delta_{n}$. По определению классов эквивалентности справедливы соотношения

$$
\begin{aligned}
& E^{\alpha}(\Pi) \cap E^{\beta}(\Pi)=\varnothing, \text { если } \alpha \neq \beta, \\
& \bigcup_{\alpha \in M} E^{\alpha}(\Pi)=\varsigma_{n} .
\end{aligned}
$$

Если обозначить через $\boldsymbol{e}^{\alpha_{i}}=\left(e^{\alpha_{1}}, \ldots, e^{\alpha_{n}}\right)$ векторы с координатами

$$
e^{\alpha}{ }_{i}{ }^{*} x_{k \sigma}, \quad \sigma \in E^{\alpha}(\Pi), \quad k \sigma=i \in \mathfrak{J}, \quad \alpha \in \mathcal{M},
$$

то $\boldsymbol{e}^{\alpha} \in{ }^{*} \Pi \subset \Pi(\alpha \in \mathcal{M})$ и формулы (8) определяют взаимно однозначное соответствие между $m$-элементными множествами $\left\{e^{\alpha}, \alpha \in \mathcal{M}\right\}$ и $\left\{E^{\alpha}(\Pi), \alpha \in \mathcal{M}\right\}$. Векторы $\boldsymbol{e}^{\alpha} \in \Pi$ называются $E$-максимальными в П.

Учитывая определение $E$-максимальных элементов и определение множества* $\subset П$, можно убедиться, что *П является выпуклой оболочкой векторов $\left\{\boldsymbol{e}^{\alpha}, \boldsymbol{\alpha} \in \mathcal{M}\right\}$. Тем самым *П будет выпуклым многогранником в $R^{+}{ }_{n}$ с вершинами $e^{\alpha}(\alpha \in \mathcal{M})$. Они же будут вершинами для полиматроида П, хотя не исчерпывают всего множества вершин П, так как, согласно условию 1), из определения 1 справедливо соотношение

$$
\Pi=\left\{x \in R^{+}{ }_{n}, x \leqslant{ }^{*} x,{ }^{*} x \in{ }^{*} \Pi\right\} .
$$

С точки зрения классификации полиматроидов $\Pi \in \Pi(n)$ оказывается существенной проблема смежности вершин многогранника *П его максимальных элементов. Напомним, что две вершины многогранника называются смежными, если они принадлежат одному и тому же ребру (одномерной грани многогранника). Следующее предложение дает ответ.на вопрос, какие вершины многогранника *П являются смежными.

Предложени е 2. Пусть задан полиматроид П $\in \Pi(n)$ с многогранником *П максимальных элементов. Для фиксированной вериины $\boldsymbol{e}^{\alpha} \in{ }^{*} \Pi$ смежными в ${ }^{*} \Pi$ будут все такие $e^{\beta} \in * \Pi$, при которых существуют число $t \in\{1, \ldots, n-1\}$ и перестановки $\sigma \in E^{\alpha}(\Pi) u \tau(t) \in E^{\beta}(\Pi)$ так, что если $\sigma=\left\{1 \sigma, \ldots, t \sigma,(t+1) \sigma, \ldots, n_{\sigma}\right\}, \quad$ то $\tau(t)=$ $=(1 \sigma, \ldots,(t+1) \sigma, t \sigma, \ldots, n \sigma)$. Нмеет место и обратное.

Для доказательства нужно иметь в виду, что в силу вышесказанного вершины *П являются одновременно и вершинами П, который задается системой (2). Следовательно, $n-1$ независимых неравенств системы (2) определяют ребро для П. Но если различные вершины $\boldsymbol{e}^{\alpha}, \boldsymbol{e}^{\beta} \in{ }^{*} \Pi \subset \Pi$ удовлетворяют условиям предложения, то эти неравенства вполне определены с помощью формул (4) и (8).

Заметим, что условия смежности вершин многогранников $* \Pi \subset \Pi \in$ $\in \Pi(n)$ очень похожи на условия смежности вершин перестановочных многогранников (см. $\left[{ }^{1}\right]$, с. 185$)$ и в случае, когда $m=n !$, они даже 
совпадают. Кроме того, очевидно, что число вершин, смежных с данной в *П, в общем случае не определено однозначно, а существенно зависит от мощностей классов эквивалентности $E^{\alpha}(\Pi) \subset \mathcal{\varepsilon}_{n}$.

\section{§ 3. Некоторые числовые характеристики полиматроидов}

3.1. Определения числовых характеристик. Теперь мы можем ввести такие числовые характеристики, с помощью которых полностью определяется комбинаторная структура $n$-мерного полиматроида $\Pi \in \Pi(n)$. Из вышесказанного ясно, что важную роль при этом играет параметр $m$ - число вершин многогранника *П, т. е. максимальных элементов полиматроида П; или, что то же самое, - число $E$-максимальных элементов полиматроида П, которое может принимать значения

$$
1 \leqslant m \leqslant n ! .
$$

Пусть значение $m \in\{1, \ldots, n !\}$ зафиксировано. Тогда каждый из $m$ различных $E$-максимальных элементов $\boldsymbol{e}^{\alpha}(\alpha \in \mathcal{M})$ полиматроида П определен по (8) однозначно некоторым классом эквивалентности перестановок $E^{\alpha}(\Pi) \subseteq \mathcal{S}_{n}(\alpha \in \mathcal{M})$. Если ввести обозначение $\left|E^{\alpha}(\Pi)\right|=p^{\alpha}$, то величина $p^{\alpha}$ является числом тех $\sigma$-максимальных элементов полиматроида П, которые склеиваются в один $E$-максимальный. При этом, учитывая свойства (7) классов эквивалентности $E^{\alpha}(\Pi)$, имеет место соотношение

$$
\sum_{\alpha \in M} p^{\alpha}=n !
$$

Не ограничивая общности, можно считать, что классы $E^{\alpha}(\Pi) \subseteq \delta_{n}$ упорядочены по убыванию их мощностей. Тогда

$$
n ! \geqslant p^{1} \geqslant \ldots \geqslant p^{m} \geqslant 1 \text {. }
$$

Отсюда видно, что числами $p^{\alpha}(\alpha \in M)$ можно выбрать упорядоченные по убыванию члены разложения числа $n$ ! на $m$ слагаемых.

Кроме мощности $p^{\alpha}$, заданный класс эквивалентности $E^{\alpha}(\Pi) \subseteq \mathbf{S}_{n}$ характеризуется несколько подробнее параметрами, описывающими входящие в него перестановки. Для этого мы воспользуемся следующими понятиями, в которых фиксированы полиматроид $\Pi \subset П(n)$, число $u \in\{1, \ldots, n-1\}$ и $(u-1)$-элементное упорядоченное подмножество $\{i(1), \ldots, i(u-1)\} \equiv J(u-1) \subset J$. При этом считаем, что если $u=1$, то $\jmath(u-1)=\varnothing$.

Определение 5. Назовем э(и-1)-надстройкой подмножества $\{i(1), \ldots, i(u-1)\} \equiv J(u-1) \subset J$ подмножество тех индексов $\alpha \in \mathcal{M}$, при которых класс $E^{\alpha}(\Pi)$ содержит хотя бы одну перестановку $\sigma=(1 \sigma, \ldots, n \sigma) \in S_{n} c 1 \sigma=i(1), \ldots,(u-1) \sigma=i(u-1)$.

Обозначим такую надстройку через $\mathfrak{M}(\mathfrak{J}(u-1)) \subseteq \mathcal{M}$. При этом естественно считать, что $\mathcal{M}(\varnothing) \equiv \mathcal{M}$.

О пределени е 6 . Пусть при фиксированном $э(u-1)=\{i(1), \ldots$ $\ldots, i(u-1)\}$ определено $\mathcal{M}(J(u-1)) \subseteq \mathcal{M} u$ задано $\alpha \in \mathcal{M}(\jmath(u-1))$.

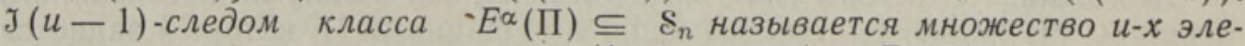
ментов тех перестановок $\sigma=(1 \sigma, \ldots, n \sigma) \in E^{\alpha}(\Pi)$, при которых $1 \sigma=i(1), \ldots,(u-1) \sigma^{\prime}=i(u-1)$.

Обозначим это множество через $\Sigma(J(u-1), \alpha)$ и назовем его мощность $\Im(u-1)$-шириной класса $E^{\alpha}(\Pi)$. Пусть $|\Sigma(J(u-1), \boldsymbol{\alpha})|=$ $=q^{\alpha}(J(u-1))$. Из определения 6 ясно, что Ј $(u-1)$-след определен однозначно для любого класса $E^{\alpha}(\Pi)$ с $\alpha \in \mathcal{M}(\Im(u-1))$. В частном случае $u=1$ мы будем говорить упрощенно о следе и ширине каждого 
класса $E^{\alpha}(\Pi), \alpha \in \mathcal{H}$, та́к как по нашему соглашению $J(0)=\varnothing$ и $\boldsymbol{M}\left(\mathcal{J}^{\prime}(0)\right) \equiv \mathcal{M}$. Введем также соответствующие сокращенные обозначения

$$
\begin{aligned}
& \Sigma(J(0), \alpha) \equiv \Sigma(\alpha)=\left\{1 \sigma, \sigma \in E^{\alpha}(\Pi)\right\}, \quad \alpha \in \mathcal{M} \\
& q^{\alpha}(J(0)) \equiv q^{\alpha}=|\Sigma(\alpha)|, \quad \alpha \in \mathcal{M} .
\end{aligned}
$$

Значения $Ј(u-1)$-ширин классов эквивалентности $E^{\alpha}(\Pi) \subseteq S_{n}$ $(u \in\{1, \ldots, n-1\}, \mathcal{J}(u-1) \subset J, \alpha \in \mathcal{M}(J(u-1)))$ являются числами, характеризующими формальную структуру всех классов $E^{\alpha}(\Pi)$, а тем самым и комбинаторную структуру соответствующего полиматронда П. Это следует из формул (4), (5), (6) и (8), с помсщью которых проводится геометрическое истолкование определенных величин. Действительно, соотношениями (8) каждому б-максимальному элементу " $\boldsymbol{x}^{\sigma} \in \Pi$ однозначно сопоставляетсл $E$-максимальный элемент $\boldsymbol{\epsilon}^{\alpha} \in \mathrm{II}$, $\alpha \in \mathcal{M}$, соответствующий такому классу эквивалентности $E^{\alpha}(\Pi)$, который содержит $\sigma \in E^{\alpha}(\Pi)$. По формулам (5) и (8) для таких $\sigma$ и $\alpha$ при $i=u \sigma(u=1, \ldots, n)$ справедливо

$$
e^{\alpha_{i}}={ }^{*} x^{\sigma} u \sigma=\max _{x \in \Pi_{(1 \ldots(u-1)) \sigma}} x_{u \sigma} .
$$

Если $1 \sigma=i(1), \ldots,(u-1) \sigma=i(u-1)$, то, обозначив $\Pi_{(1 \ldots(u-1))_{\sigma}}=$ $=\Pi(\jmath(u-1))$ и следуя определению 6 , очевидно, что во множество $\Sigma(J(u-1), \alpha)$ входят индексы $u \sigma \in J$ тех координат $e^{\alpha}{ }_{i}$ максимального элемента $\boldsymbol{e}^{\alpha} \in \Pi$, которые принимают максимальные возможные значения в П(J(u-1)). Иными словами, J(u-1)-ширина $q^{\alpha}(\Im(u-1))$ класса $E^{\alpha}(\Pi) \subseteq \mathcal{S}_{n}$ с $\alpha \in \mathcal{M}(J(u-1))$ указывает число тех координат вектора $\boldsymbol{e}^{\alpha} \in \Pi, \quad$ которые принимают максимальные значения в $\Pi(J(u-1))$, что в свою очередь вполне определено граневой структурой полиматронда П.

3.2. Теоремы об оценках введенных характеристик. Как мы увидим немного позже, комплекты параметров $m, p^{\alpha}, q^{\alpha}(\jmath(u-1))$, связанные с произвольным полиматроидом $\Pi \in \Pi(n)$, могут служить для получения их классификаций, удовлетворяющих условиям из $\$ 1$. Но в связи с этим целесообразно остановиться на проблемах оценки этих параметров. Следует же заметить, что предлагаемые результаты не претендуют на исчерпанность проблемы, а являются лишь первым шагом на этом пути. Результаты формулируются -в теоремах, доказательства которых опускаются для краткости изложения.

Для значений $m$ и $p^{\alpha}(\alpha \in \mathcal{M})$ некоторые оценки в виде (9) и (11) уже имеются. Но они уточняются следующими утверждениями.

Те орем а 4. Пусть П является п-мерным полиматроидом. Равенство $m_{i}=1$ справедливо тогда и только тогда, когда соответствующий след $\Sigma(\alpha)(\alpha \in \mathcal{M}=\{1, \ldots, m\})$ совпадает с множеством $J_{1}=\{1, \ldots, n\}$ Теорем а 5. Пусть П является п-мерным полиматроидом с фиксированным значеншем $m \in\{1, \ldots, n !\}$. Тогда параметры $p^{\alpha}=\left|E^{\alpha}(\Pi)\right|$ $(\alpha \in \mathcal{M})$ удовлетворяют, кроме соотношения $\sum_{\alpha \in M} p^{\alpha}=n !$, следующим утверждениям.

1. Если $m:=1$, то $p^{1}=n$ !.

2. Если $1<m \leqslant n$, то $1 \leqslant p^{m} \leqslant \ldots \leqslant p^{1} \leqslant(n-1)(n-1)$ !. Нмеет место и обратное: если $p^{1}=n !$, то $m=1$ и если $p^{1} \leqslant$ $\leqslant(n-1)(n-1)$ !, ,о $m>1$.

Перейдем к нахождению оценки значений $q^{\alpha}(\jmath(u-1))$ для произвольного полиматронда $\Pi \in \Pi(n)$. Пусть $u \in\{1, \ldots, n-1\}$, 
$J(u-1) \subset \Im$ и $\alpha \in M(J(u-1))$. Тогда с учетом (8), (4) и (5) из определения 5 следует, что $\boldsymbol{e}^{\alpha} \in \Pi(\Im(u-1)) \subset \Pi$. Тем самым из $n$ координат вектора $\boldsymbol{e}^{\alpha} \in \Pi \subset R^{+}{ }_{n}$ фиксированы $u-1$. И если при $\sigma \in E^{\alpha}(\Pi)$ имеет место $1 \sigma=i(1), \ldots,(u-1) \sigma=i(u-1), \quad$ то ими будут $e^{\alpha}{ }_{i(1)}={ }^{*} x^{\sigma}{ }_{1 \sigma}, \ldots, e^{\alpha}{ }_{i(u-1)}={ }^{*} x^{\sigma}{ }_{(u-1) \sigma}$. Но тогда у перестановки $\sigma \in E^{\alpha}(\Pi)$ $u \sigma=i \in J$.не может принимать более чем $n-(u-1)$ значений, входящих в ЈЈ(u-1). Отсюда следует довольно грубая оценка значения $\jmath(u-1)$-ширины класса $E^{\alpha}($ I) . А именно, при заданных $u \in$ $\in\{1, \ldots, n-1\}, J(u-1) \subset J$ и $\alpha \in \mathcal{M}(J(u-1))$ имеет место

$$
1 \leqslant q^{\alpha}(J(u-1)) \leqslant \min \left(p^{\alpha}, n-u+1\right) .
$$

В частном случае $u=1$ эта оценка несколько уточняется. А именно, справедлива

Те орем а 6. Пусть П является п-мерным полиматроидом с фиксированным значением $m \in\{1, \ldots, n !\}$. Тогда при заданном $\alpha \in \mathcal{M}$ ширина $q^{\alpha}$ класса $E^{\alpha}(\Pi) \subseteq \delta_{n}$ может принимать только значения, удовлетворяющие следующим условиям.

1. Если $1 \leqslant p^{\alpha} \leqslant(n-1)$ !, то $1 \leqslant q^{\alpha} \leqslant \min \left(p^{\alpha}, n-1\right)$.

2. Eсли $(n-1) !<p^{\alpha} \leqslant(n-1)(n-1)$ !, то $2 \leqslant q^{\alpha} \leqslant n-1$.

3. Если $p^{1}=n !$, то $q^{1}=n$.

Перснумерацией классов эквивалентности всегда можно добиться того, что если $\alpha(1)<\ldots<\alpha(t),\{\alpha(1), \ldots, \alpha(t)\} \subseteq \mathcal{M}$ и $p^{\alpha(1)}=\ldots$ $\ldots=p^{\alpha(t)}$, то имеют место $q^{\alpha(1)} \geqslant \ldots \geqslant q^{\alpha(t)}$.

\section{3. Об условиях эквивалентности перестановок относительно поли-} матроида. Чтобы использовать введенные характеристики как параметры классов некоторой классификации полиматроидов, нужно знать, какие элементы множества перестановок $S_{n}$ вообще могут оказаться эквивалентными относительно некоторого $n$-мерного полиматроида П. Такие условия получены нами с помощью условий смежности вершин многогранника максимальных элементов *П СП из предложения '2.

Пусть фиксирована перестановка $\sigma=(1 \sigma, \ldots, n \sigma) \in \mathcal{S}_{n}$ и пусть $s \in\{1, \ldots, n-1\}$. Введем обозначение

$S_{n}(\sigma)=\{\tau(s)=(1 \sigma, \ldots,(s+1) \sigma, s \sigma, \ldots, n \sigma), s=1, \ldots, n-1\} \subset S_{n}$

Если считать в обозначениях $\S 1$, что $\mathfrak{x}_{\sigma}^{0}=\varnothing$ при любом $\sigma \in S_{n}$, то с учетом определений 2 и 4 получается

Теорем а 7. Перестановки $\sigma \in S_{n} u \tau(s) \in S_{n}(\sigma)$ өквивалентны относительно полиматроида $\Pi \in \Pi(n)$, заданного системой (2), тогда и только тогда, когда в этой системе

$$
r\left(X_{\sigma}^{s}\right)+r\left(X_{\tau(s)}^{s}\right)=r\left(X_{\sigma-1}^{s}\right)+r\left(X^{s+1} \sigma\right) .
$$

Что касается произвольных перестановок $\sigma, \tau \in S_{n}$, то, в силу транзитивности отношения 'эквивалентности, для них справедливо

П р едл о же н и е 3. Пусть П является некоторым п-мерным полиматроидом. Если для перестановок $\sigma, \tau \in \mathcal{S}_{n}$ существует хотя бы одна последовательность таких параметров $s(1), \ldots, s(v) \in\{1, \ldots, n-1\}$ $(v \geqslant 1), \quad$ что $\mathrm{Q}^{1}(s(1)) \in \mathrm{S}_{n}(\sigma), \ldots, \tau=\mathrm{Q}^{v}(s(v)) \in \mathrm{S}_{n}\left(\mathrm{Q}^{v-1}(s(v-1))\right)$ и $\sigma \approx \mathrm{Q}^{1}(s(1)), \ldots, \mathrm{Q}^{v-1}(s(v-1)) \approx \tau$, то $\sigma \approx \tau$.

Выдвигается гипотеза, что последнее условие является и необходимым для эквивалентности перестановок $\sigma, \tau \in \mathcal{S}_{n}$ в смысле определения 4. Соответствующий результат получен нами в случае $n=3$ (см. раздел 5.2). 


\section{$\S$ 4. Классификация полиматроидов}

4.1. Общие принципы. В силу вышесказанного, с любым полиматрондом $\Pi \in \Pi(n)$ связан его многогранник максимальных элементов ${ }^{*} \Pi \subset \Pi$, имеющий $m$ вершин $\boldsymbol{e}^{\alpha}(\alpha \in \mathcal{M})$. Последние находятся по формулам (8) во взаимно однозначном соответствии с классами эквивалентности $E^{\alpha}(\Pi) \subseteq \delta_{n}$ перестановок в смысле определения 4. При этом классы $E^{\alpha}(\Pi)$ образуются с учетом результатов предыдущего раздела 3.3, а их формальная структура описывается множеством параметров $p^{\alpha}, q^{\alpha}(J(u-1))(\alpha \in \mathcal{M}, u \in\{1, \ldots, n-1\}, J(u-1) \subset J)$, введенных в разделе 3.1. Возможные значения для них указаны в разделе 3.2. Отсюда следует, что каждому полиматронду $\Pi \in \Pi(n)$ соответствует вполне определенный набор параметров $\left(m ; p^{\alpha} ; q^{\alpha}(\Im(u-1)), \alpha \in \mathcal{M}\right.$ $u=1, \ldots, n-1)$. Поэтому наборы чисел $\left(m ; p^{\alpha} ; q^{\alpha}(\Im(u-1)) \mid u=\right.$ $=1, \ldots, n-1)$, удовлетворяющих условиям раздела 3.2 , можно рассматривать как параметры некоторых классов $n$-мерных полиматроидов и можно задать вопрос, удовлетворяет ли такая классификация условиям $\S 1$. Кроме того, нужно выяснить, каким наборам параметров соответствуют непустые классы полиматрондов. Если эти задачи решены, то получена одна из возможных классификаций полиматроидов.

Қак было упомянуто в $\S 1$, для получения классификации полиматроидов нужно на множестве $\Pi(n)$ задать некоторое отношение эквивалентности. Если ставить целью описать соответствующие классы эквивалентности с помощью наборов параметров $\left(m ; p^{\alpha} ; q^{\alpha}(\mathfrak{\jmath}(u-1)) \mid u=\right.$ $=1, \ldots, n-1)$, то естественно требовать, чтобы при эквивалентных полиматроидах $\Pi \subset \Pi(n)$ эти наборы совпадали. Рассмотрим для примера одно из возможных определений отношения эквивалентности на множестве $\Pi(n)$. Назовем его структурной эквивалситностью, потому что оно приводит к одному из этапов на пути полного совпадения граневой структуры полиматроидов.

4.2. Структурная классификация. Пусть любой полиматроид $\Pi \in \Pi(n)$ описывается кроме параметров $m \in\{1, \ldots, n !\}$ и $p^{\alpha}(\alpha \in \mathcal{M})$, удовлетворяющих условиям теоремы 5 , еще своими ширинами $q^{\alpha}(u=1$, $\Im(0)=\varnothing)$, определенными по формулам (12) и удовлетворяющими условиям теоремы 6 . Таким образом, каждому полиматроиду П сопоставляется набор чисел $m ; p^{\alpha} ; q^{\alpha}(\alpha \in \mathcal{M})$, удовлетворяющих вышеупомянутым условиям.

О пределени е 7. Полиматроиды $\Pi, \Pi^{\prime} \in \Pi(n)$ называются структурно эквивалентными тогда и только тогда, когда для характеризующих их наборов параметров $\left(m ; p^{1}, \ldots, p^{m} ; q^{1}, \ldots, q^{m}\right)$ и $\left(m^{\prime} ; p^{1^{\prime}}, \ldots\right.$ $\left.\ldots, p^{m^{\prime}} ; q^{1^{\prime}}, \ldots, q^{m^{\prime}}\right)$ справедливы равенства $m=m^{\prime}, p^{1}=p^{1^{\prime}}, \ldots, p^{m}=$ $=p^{m^{\prime}}, q^{1}=q^{1^{\prime}}, \ldots, q^{m}=q^{m^{\prime}}$ соответственно.

Если $n$-мерные полиматропды структурно эквивалентны, то обозначим $\Pi \sim \Pi^{\prime}$. Определенное отношение является эквивалентностью, так как оно рефлексивно $(\Pi \sim \Pi)$, симметрично (если $\Pi \sim \Pi^{\prime}$, то $\left.\Pi^{\prime} \sim \Pi\right)$ и транзитивно (если $\Pi \sim \Pi^{\prime}$ и $\Pi^{\prime} \sim \Pi^{\prime \prime}$, то $\left.\Pi \sim \Pi^{\prime \prime}\right)$. Обозначим соответствующие классы эквивалентности кратко через $L\left(m ; p^{\alpha} ; q^{\alpha}\right) \subset$ $\subset \Pi(n)$. Полученная классификация называется структурной классификацией $n$-мерных полиматрондов.

Используя определение 3 и предложение 1 , получается

Предложе н и е 4. Если полиматроид $\Pi^{\prime} \in \Pi(n)$ получается из полиматроида $\Pi \in \Pi(n)$ либо положительной гомотетией пространства $R_{n}$, либо перестановкой координатных осей, то П и П' структурно эквивалентны.

Из этого предложения и простого рассуждения следует, что опреде- 
ленная нами структурная классификация удовлетворяет требованиям $\$ 1$, т. е. справедливо

Предложени е 5. Структурная классификация n-мерных полиматроидов является конечной, приводимой и гомотетичной.

Заметим, что при $u>1$ аналогичным путем получаются различные более точные классификации полиматроидов, а при $u=n-1$ каждый класс эквивалентности в П $(n)$ содержит гомотетичные полиматроиды, граневая структура которых определена с точностью до его расположения относительно координатных осей.

Нахождение всех классов структурной классификации $n$-мерных полиматроидов целесообразно разбить на два этапа.

1. Определение всех допустимых в смысле теорем 4, 5 и 6 наборов параметров $\left(m ; p^{\alpha} ; q^{\alpha}, \alpha \in \mathcal{M}\right)$ классов $L\left(m ; p^{\alpha}, q^{\alpha}\right) \subset \Pi(n)$ структурной классификации. По результатам $\S 3$ ясно, что для этого нужно сперва перечислить всевозможные разложения числа $n !$ на $m(1 \leqslant m \leqslant n !)$ невозрастающих положительных целочисленных слагаемых, потом для каждого разложения проверить условия теоремы 5 , а затем для разложений, удовлетворяющих упомянутым условиям, по теореме 6 определить всевозможные значения параметров $q^{\alpha}(\alpha \in \mathcal{A})$. Получаемые таким образом наборы $\left(m ; p^{\alpha} ; q^{\alpha}(\alpha \in \mathcal{M})\right)$ составляют искомый список допустимых наборов параметров структурной классификации $n$-мерных полиматроидов.

2. Решение проблемы непустоты класса $L\left(m ; p^{\alpha} ; q^{\alpha}\right) \subset \Pi(n)$ для всех допустимых наборов параметров $\left(m ; p^{\alpha} ; q^{\alpha}(\alpha \in \mathcal{M})\right)$. Так как по теореме 2 любой полиматроид задается системой (2), то поставленная проблема будет решена положительно, если для фиксированного допустимого набора $\left(m ; p^{\alpha} ; q^{\alpha}(\alpha \in \mathcal{M})\right)$ параметров будет указана хотя бы одна система вида (2), задающая полиматронд $\Pi \in \Pi(n)$ с таким набором параметров. Если же такой системы не существует, то будем считать, что соответствующий класс $L\left(m ; p^{\alpha} ; q^{\alpha}\right)$ пуст и что набор $\left(m ; p^{\alpha} ; q^{\alpha}(\alpha \in \mathcal{M})\right)$ не служит набором параметров для $n$-мерных полиматрондов.

Покажем на примере трехмерных полиматроидов, как найти те наборы параметров $\left(m ; p^{\alpha} ; q^{\alpha}(\alpha \in \mathcal{M})\right)$ их структурной классификации, которым соответствуют непустые классы $L\left(m ; p^{\alpha} ; q^{\alpha}\right) \subset \Pi(3)$. По теореме 2 любой трехмерный полиматроид $\Pi \in \Pi(3)$ задается системой (2) линейных неравенств, в правых частях которых стоят значения некоторой неубывающей субмодулярной функции над трехэлементным множеством $J=\{1,2,3\}$. Поэтому, прежде чем перейти к нахождению структурных классов $L\left(m ; p^{\alpha} ; q^{\alpha}\right) \subset \Pi(3)$, целесообразно сперва несколько подробнее изучить свойства таких функций. Так как по предложению 5 структурная классификация приводима, т. е. классы $L\left(m ; p^{\alpha} ; q^{\alpha}\right)$ определены с точностью до перенумерации координатных осей, то предположим в дальнейшем, что $J=\{1,2,3\}=\{i, j, k\}$.

\section{$\S 5$. Трехмерные полиматроиды}

5.1. Условия субмодулярности функции над трехэлементным множеством. Если $J=\{i, j, k\}$, то $2(J)=\{\varnothing,\{i\},\{j\},\{k\},\{i, j\},\{i, k\},\{j, k\}$, $\{i, j, k\}\}$. Пусть $r: 2(\mathfrak{J}) \rightarrow R$ является неубывающей субмодулярной функцией над $⿱$ и $r(\varnothing)=0$. Обозначим

$$
\begin{array}{lll}
r(\{i\})=r_{i}, & r(\{j\})=r_{j}, & r(\{k\})=r_{k}, \\
r(\{i, j\})=r_{i j}, & r(\{i, k\})=r_{i k}, & r(\{j, k\})=r_{j k}, \\
r(\{i, j, k\})=r . &
\end{array}
$$


Так как аргументы функции $r: 2(\jmath) \rightarrow R$ суть неупорядоченные подмножества, то она симметрична относительно любой перестановки элементов внутри своего аргумента, в том числе и

$$
r_{i j}=r_{j i}, \quad r_{i k}=r_{k i}, \quad r_{j k}=r_{k j} .
$$

В новых обозначениях условия (1) субмодулярности функции $r: 2(\jmath) \rightarrow R$ принимают специальный вид. А именно, имеет место

Предложение 6 . Если функция $r: 2(\jmath) \rightarrow R$ является субмодулярной и $|\jmath|=3$, то ее действительные значения удовлетворяют условиям

$$
\begin{aligned}
& r_{a b} \leqslant r_{a}+r_{b} \quad \text { для любых } a, b \in J, a \neq b ; \\
& r \leqslant r_{a}+r_{b c} \quad \text { длякаждого } a \in J \text { при }\{a, b, c\}=J \text {; } \\
& r \leqslant r_{a b}+r_{a c}-r_{a} \text { для каждого } a \in J \text { nрu }\{a, b, c\}=J .
\end{aligned}
$$

Доказательство получается исходя из предположения субмодулярности функции $r: 2(\jmath) \rightarrow R$, если в условиях (1) рассматривать отдельно все нетривиальные возможности $\mathfrak{x} \neq \mathcal{y}$ при $\mathfrak{X}, \mathcal{Y} \in 2(\mathfrak{J})$. Ими будут

1. $\mathfrak{X}=\{a\}, \quad y=\{b\}, \quad$ где $a, b \in \mathfrak{J}, a \neq b$;

2. $x=\{a\}, \quad$ У $=\{b, c\}$, при любом $a \in J$ и $\{a, b, c\}=\mathfrak{J}$;

3. $\mathfrak{X}=\{a, b\}, y=\{a, c\}$, при любом $a \in \mathcal{J}$ и $\{a, b, c\}=J$.

Подробности опускаются и предложение считается доказанным.

Необходимые условия (15) субмодулярности функции $r: 2(\jmath) \rightarrow R$ при $|\jmath|=3$ зависимы между собой. Основываясь на них, доказывается

Предложение 7. Если $|J|=3$, то неубывающая функция $r: 2(\jmath) \rightarrow R$ субмодулярна тогда и только тогда, когда ее действительные значения удовлетворяют одному из четырех комплектов условий (в табл. 1; ими исчерпаны все возможности).

Для доказательства необходимости условий заметим, что неупорядоченную пару $a, b \in J, a \neq b$, можно выбрать тремя способами и поэтому первое условие в (15) фактически включает три условия (табл. 1), занимающие четыре строки, так как с точностью до перенумерации элементов множества $J$ существуют четыре различные комбинации знаков равенств и неравенств в соответствующих условиях (15) и они исчерпывают все возможности. Чтобы закончить доказательство необходимости условий табл. 1, следует в каждом из этих четырех случаев выяснить наиболее сильное из шести возможных ограничений для $r$, заданпых условиями (15). Они указаны в последнем столбце табл. 1, из условий которой простыми вычислениями и получается достаточность.

Заметим лишь, что фактически табл. 1 содержит 10 типов условий субмодулярности функции $r: 2(\mathfrak{J}) \rightarrow R$ для $|\mathfrak{J}|=3$, так как в трех

Таблица 1

Условия субмодулярности функции $r: 2($ 孔) $\rightarrow R$ при $\mid$ J $\mid=3$

\begin{tabular}{r|c|c|c|c}
\hline $\begin{array}{r}\text { Комплект } \\
\text { условий }\end{array}$ & Условие для $r_{i j}$ & Условие для $r_{i h}$ & Условие для $r_{j h}$ & Условие для $r$ \\
\hline
\end{tabular}

$\begin{array}{lllll}1 & =r_{i}+r_{j} & =r_{i}+r_{k} & =r_{j}+r_{h} & \leqslant r_{i}+r_{j}+r_{h} \\ 2 & =r_{i}+r_{j} & =r_{i}+r_{k} & <r_{j}+r_{h} & \leqslant r_{i}+r_{j k} \\ 3 & =r_{i}+r_{j} & <r_{i}+r_{h} & <r_{j}+r_{h} & \leqslant r_{i k}+r_{j k}-r_{h} \\ 4 & <r_{i}+r_{j} & <r_{i}+r_{k} & <r_{j}+r_{k} & \leqslant r_{a b}+r_{a c}-r_{a}\end{array}$


первых строках последнего столбцца содержится по два, а в четвертом четыре типа ограничений для $r$ с одинаковыми ограничениями на $r_{a b}$, $a, b \in J, a \neq b$.

5.2. Задание трехмерных полиматроидов. Результаты первых двух предложений настоящего раздела не требуют специального доказательства, так как они являются лишь частными случаями теорем 2 и 3. А именно, с учетом предложения 7 теорему 2 можно переформулировать следующим образом.

Предл ожен и е 8 . Многогранник $\Pi \subset R+_{3}$ является трехмерным полиматроидом тогда и только тогда, когда он задается в $\mathrm{R}_{3}$ системой линейных неравенств

$$
\begin{aligned}
& 0 \leqslant x_{a} \leqslant r_{a}, a \in J=\{i, j, k\}=\{1,2,3\}, \\
& x_{a}+x_{b} \leqslant r_{a b}, a, b \in J, a<b, \\
& x_{a}+x_{b}+x_{c} \leqslant r, \quad\{a, b, c\}=J
\end{aligned}
$$

действительные параметры $r_{a}>0, r_{a b}=r_{\text {bа }}>0$ и $r>0$ которой удовлетворяют одному из комплектов условий табл. 1.

Если полиматроид $\Pi \in \Pi(3)$ задан системой (16), то нам удобно пользоваться значениями координат $\left({ }^{*} x^{\sigma}{ }_{i},{ }^{*} x^{\sigma}{ }_{j},{ }^{*} x^{\sigma_{k}}\right)$ произвольного $\sigma$-максимального элемента ${ }^{*} \boldsymbol{x}^{\sigma} \in{ }^{*} \Pi \subset \Pi\left(\sigma \in \boldsymbol{S}_{3}\right)$ этого полиматроида. Как координаты специальных вершин многогранника $\Pi \subset R^{+}{ }_{3}$ они по теореме 3 и формулам (4) вполне определены системой (16).

Предложени е 9. Пусть $\sigma \in \mathrm{S}_{3}=\{(i, j, k),(i, k, j),(j, i, k),(j, k, i)$, $(k, i, j),(k, j, i)\}$. Тогда б-максимальными элементами полиматроида $\Pi \in \Pi(3)$, заданного системой (16), будут векторы ${ }^{*} \boldsymbol{x}^{\sigma} \in R_{3} c$ координатами $\left({ }^{*} x^{\sigma}{ }_{i},{ }^{*} x^{\sigma_{j}},{ }^{*} x^{\sigma_{k}}\right)$, значения которых представлены в табл. 2.

Напомним, что положительные числа $r_{a}, r_{a b}, r \in R$ в этой таблице удовлетворяют одному из 10 комплектов условий из табл. 1 , при которых (16) задает некоторый полиматроид П $\in \Pi(3)$. Поэтому в каждом из этих случаев координаты ${ }^{*} x^{\sigma_{i}},{ }^{*} x^{\sigma}{ }_{j},{ }^{*} x^{\sigma_{k}}\left(\sigma \in S_{3}\right)$ принимают вполне определенные значения. Но по определению 4 перестановки $\sigma, \tau \in S_{3}$ называются эквивалентными относительно рассматриваемого полимат-

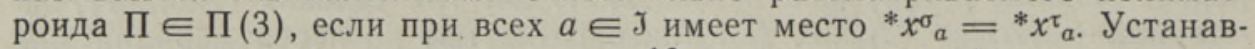
ливая все такие равенства во всех 10 случаях условий на параметры $r_{a}, r_{a b}, r$ системы (16), получаются классы эквивалентности $E^{\alpha}(\Pi) \subseteq \mathcal{S}_{3}$ перестановок относительно соответствующего полиматроида. Опуская вычисления, представим получаемые результаты в табл. 3, сохраняя в номере разбиения номер условий из табл. 1.

С помощью этой таблицы легко доказывается

Т е о р е а 8. Пусть П является трехмерным полиматроидом. Две перестановки $\sigma \in S_{3}, \tau \notin S_{3}(\sigma)$ будут эквивалентными относительно поли-

Таблица 2

Координаты б-максимальных элементов $\Pi \in \Pi(3)$

\begin{tabular}{c|c|c|c}
\hline $\begin{array}{c}\text { Перестановка } \\
\sigma \in \mathrm{S}_{3}\end{array}$ & ${ }^{*} x_{i} \sigma$ & ${ }^{*} x_{j} \sigma$ & ${ }^{*} x_{h} \sigma$ \\
\hline$(i, j, k)$ & $r_{i}$ & $r_{i j}-r_{i}$ & $r-r_{i j}$ \\
$(i, k, j)$ & $r_{i}$ & $r-r_{i k}$ & $r_{i k}-r_{i}$ \\
$(j, i, k)$ & $r_{i j}-r_{j}$ & $r_{j}$ & $r-r_{j i}$ \\
$(j, k, i)$ & $r-r_{j k}$ & $r_{j}$ & $r_{j k}-r_{j}$ \\
$(k, i, j)$ & $r_{i k}-r_{k}$ & $r-r_{i k}$ & $r_{k}$ \\
$(k, j, i)$ & $r-r_{j k}$ & $r_{j k}-r_{k}$ &
\end{tabular}


матроида П тогда и только тогда, когда существует хотя бы одна последовательность параметров $s(1), \ldots, s(v) \in\{1,2\} \quad(v \geqslant 1)$ так, что $\varrho^{1}(s(1)) \in S_{3}(\sigma), \ldots, \tau=\varrho^{v}(s(v)) \in S_{3}\left(\varrho^{v-1}(s(v-1))\right) u \sigma \approx \varrho^{1}(s(1)), \ldots$ $\ldots, \varrho^{v-1}(s(v-1)) \approx \tau$.

Достаточность условий теоремы является следствием транзитивности отношения эквивалентности так же, как при предложении 3 , частным случаем которого она является.

Как видно из табл. 3, необходимость условий предложения нужно доказать лишь для случаев $1 \mathrm{~A}$ и $2 \mathrm{~A}$, так как остальные относятся к теореме 7 . Тем самым предположим, что $\sigma \approx \tau$ и $\tau \notin S_{3}(\sigma)$. Нужно указать хотя бы одну последовательность параметров $s(1), \ldots, s(v)$, удовлетворяющую условиям теоремы. Например, если $\sigma=(i, j, k)$ и $\tau=(j, k, i)$, то при $s(1)=1, s(2)=2$ будем, в силу (14), иметь $\mathrm{Q}^{1}(s(1))=(j, i, k), \varrho^{2}(s(2))=(j, k, i)=\tau$, где как в случае $1 \mathrm{~A}$, так и в случае 2 А справедливо $\sigma \approx \mathrm{Q}^{1}(1), \mathrm{Q}^{1}(1) \approx \tau$, что и требовалось доказать. Следует заметить, что для рассматриваемой пары $\sigma, \tau \in \delta_{3}$ в случае $2 \mathrm{~A}$ последовательность $s(1), \ldots, s(v)$ определена однозначно, а в случае $1 \mathrm{~A}$ таких последовательностей больше чем одна. Аналогичным образом искомые последовательности параметров получаются для всех других пар $\sigma \approx \tau, \tau \notin \delta_{3}(\sigma)$. Этим можно считать предложение доказанным.

таблица 3

Всевозможные разбиения множества $S_{3}$ на классы $E \alpha(\Pi)$

\begin{tabular}{c|l|l|l|l|l}
\hline $\begin{array}{c}\text { Номер } \\
\text { разбие- } \\
\text { ния }\end{array}$ & $\begin{array}{l}\text { Условне } \\
\text { для } r_{i j}\end{array}$ & $\begin{array}{l}\text { Условие } \\
\text { для } r_{i h}\end{array}$ & $\begin{array}{l}\text { Условие } \\
\text { для } r_{j k}\end{array}$ & Условие для $r$ & Классы $E \alpha(\Pi)$ \\
\hline
\end{tabular}

$1 \mathrm{~A}$

15

$$
=r_{i}+r_{j}=r_{i}+r_{k}=r_{j}+r_{k}=r_{i}+r_{j}+r_{k}
$$

$=\quad<r_{i}+r_{j}+r_{k}$

$\mathrm{S}_{3}$

$\{(i, j, k),(j, i, k)\}$, $\left\{\begin{array}{l}(i, k, j),(k, i, j)\} \\ (j, k, i),(k, j, i)\}\end{array}\right.$

$2 \mathrm{~A}$

$$
=r_{i}+r_{j}=r_{i}+r_{k}<r_{j}+r_{k}=r_{i}+r_{j k}
$$$$
" \quad<r_{i}+r_{j k}
$$

2Б

"

"

$" \quad<r_{i}+r_{j k}$

$\{(i, j, k),(j, i, k)\}$,

$$
=r_{i}+r_{j}<r_{i}+r_{k}<r_{j}+r_{k}=r_{i k}+r_{j h}-r_{k}
$$

$\{(i, j, k),(j, i, k),(j, k, i)\}$, $\{(i, k, j),(k, i, j),(k, j, i)\}$

$$
\text { " } \quad \text { " }
$$$$
\text { " } \quad<r_{i k}+r_{j k}-r_{h}
$$
$\{(i, k, j),(k, i, j)\}$, $\{(j, k, i)\},\{(k, j, i)\}$

$4 \mathrm{~A}$ $<r_{i}+r_{j}<r_{i}+r_{k}<r_{j}+r_{k}=r_{a b}+r_{a c}-r_{a}$ для любого $a \in J$

$=r_{i j}+r_{i k}-r_{i},\{(i, j, k),(i, k, j)\}$, $=r_{i j}+r_{j k}-r_{j}\{(j, i, k),(j, k, i)\}$, $<r_{i k}+r_{j k}-r_{k}\{(k, i, j)\},\{(k, j, i)\}$ 


\section{§ 6. Структурная классификация трехмерных полиматроидов}

6.1. Множество допустимых параметров. По $§ 4$ задача структурной классификации трехмерных полиматроидов решается в двух этапах. На первом этапе ищется список всевозможных допустимых в смысле теорем 5 и 6 наборов параметров $\left(m ; p^{\alpha} ; q^{\alpha}(\alpha \in \mathcal{M})\right)$. Так как теперь $n:=3$, то $n !=6$ и $(n-1)(n-1) !=4$. Следовательно, условиями, налагаемыми теоремами 5 и 6 на параметры $m, p^{\alpha}, q^{\alpha}(\alpha \in \mathcal{M})$ будут

$$
\begin{aligned}
& m \in\{1, \ldots, 6\}, \\
& \sum_{\alpha \in M} p^{\alpha}=6, \\
& p^{1}=6, \text { если } m=1, \\
& 1 \leqslant p^{m} \leqslant \ldots \leqslant p^{1} \leqslant 4, \text { если } m \in\{2, \ldots, 6\}, \\
& \text { если } p^{\alpha}=1 \text {, то } q^{\alpha}=1, \\
& \text { если } p^{\alpha}=2, \text { то } q^{\alpha} \in\{1,2\}, \\
& \text { если } p^{\alpha} \in\{3,4\}, \text { то } q^{\alpha}=2, \\
& \text { если } p^{\alpha}=6, \text { то } q^{\alpha}=3 .
\end{aligned}
$$

Простая проверка показывает, что число 6 разлагается на невозрастающие положительные целочисленные слагаемые 11 способами. А именно:

$6=6,6=5+1,6=4+2,6=3+3,6=4+1+1,6=3+2+1,6=2+2+2 ;$ $6=3+1+1+1,6=2+2+1+1,6=2+1+1+1+1,6=1+1+1+1+1+1$.

Среди этих разложений условиям (17) не удовлетворяет лишь разложение $6=5+1$, так как $m=2$, но $p^{1}=5>4$. Если теперь учитывать замечание из $\S 3$ и определить значения $q^{\alpha}$ соответственно значениям $p^{\alpha}$ из условий (17), то получается

Предложени е 10. Допустимыми наборами параметров ( $m ; p^{\alpha}$; $\left.q^{\alpha}(\alpha \in \mathbb{M})\right)$ структурной классификации трехмерных полиматроидов являются

1. $(1: 6 ; 3)$

2. $(2 ; 4,2 ; 2,2)$

3. $(2 ; 4,2 ; 2,1)$

4. $(2 ; 3,3 ; 2,2)$

5. $(3 ; 4,1,1 ; 2,1,1)$

6. $(3 ; 3,2,1 ; 2,2,1)$
7. $(3 ; 3,2,1 ; 2,1,1)$

8. $(3 ; 2,2,2 ; 2,2,2)$

9. $(3 ; 2,2,2 ; 2,2,1)$

10. $(3 ; 2,2,2 ; 2,1,1)$

11. $(3 ; 2,2,2 ; 1,1,1)$

12. $(4 ; 3,1,1,1 ; 2,1,1,1)$
$13(4 ; 2,2,1,1 ; 2,2,1,1)$

14. $(4 ; 2,2,1,1 ; 2,1,1,1)$

15. $(4 ; 2,2,1,1 ; 1,1,1,1)$

16. $(5 ; 2,1,1,1,1 ; 2,1,1,1,1)$

17. $(5 ; 2,1,1,1,1 ; 1,1,1,1,1)$

18. $(6 ; 1,1,1,1,1,1 ; 1,1,1,1,1,1)$

Остается выяснить, какие из упомянутых допустимых наборов могут служить наборами параметров классов структурной классификации трехмерных полиматроидов и какие не могут. Это делается на втором этапе решения задачи классификации.

6.2. Классы структурной классификации. Как уже было сказано в $\S 4$, для того, чтобы доказать непустоту класса $L\left(m ; p^{\alpha} ; q^{\alpha}\right) \subset \Pi(3)$ при некотором конкретном допустимом наборе параметров $\left(m ; p^{\alpha}\right.$; $\left.q^{\alpha}(\alpha \in \mathcal{M})\right)$ из предложения 10 , нужно указать систему (16), задающую полиматроид П из класса $L\left(m ; p^{\alpha} ; q^{\alpha}\right)$. Или, точнее, следует указать хотя бы один набор условий из табл. 1, при котором система (16) определяет полиматроид $\Pi \in \Pi(3)$ из этого класса. Наши исследования показывают, что из 18 допустимых наборов, указанных в предложении 10, существенными являются лишь 10 . Остальные же не могут служить параметрами $L\left(m ; p^{\alpha} ; q^{\alpha}\right) \subset \Pi(3)$.

Полное доказательство основного результата настоящего параграфа требует доказательства многих предложений. Для краткости сформули- 
руем одно из этих предложений и ограничимся лишь общей характеристикой хода доказательства, который одинаков во всех случаях. Пусть задан полиматроид $\Pi \in \Pi(3)$ из класса $L\left(m ; p^{\alpha} ; q^{\alpha}\right)$ с фиксированным допустимым набором параметров $\left(m ; p^{\alpha} ; q^{\alpha}(\alpha \in \mathcal{M})\right)$. Нами ищется набор условий из табл. 1, при которых система (16) задает рассматриваемый полиматроид.

Если набор $\left(m ; p^{\alpha} ; q^{\alpha}(\alpha \in M)\right)$ известен, то с учетом теорем 7 и 8 определяются классы эквивалентности $E^{\alpha}(\Pi)(\alpha \in \mathcal{M})$ с соответствующими характеристиками $p^{\alpha}, q^{\alpha}(\alpha \in \mathcal{M})$. Эти классы являются во взаимно-однозначном соответствии с $E$-максимальными элементами $\boldsymbol{e}^{\alpha}(\alpha \in \mathcal{M})$, которые однозначно сопоставлены любому $\sigma$-максимальному элементу * $\boldsymbol{x}^{\sigma}\left(\sigma \in \mathcal{\varepsilon}_{3}\right)$ формулами (8). Обозначим это соответствие через $f\left({ }^{*} \boldsymbol{x}^{\sigma}\right)=\boldsymbol{e}^{\alpha}, \sigma \in E^{\alpha}(\Pi)$. Если $\sigma \neq \tau$, то при $f\left({ }^{*} \boldsymbol{x}^{\tau}\right)=\boldsymbol{e}^{\beta}$ существует две возможности: либо $\boldsymbol{e}^{\alpha}=\boldsymbol{e}^{\beta}$, либо $\boldsymbol{e}^{\alpha} \neq \boldsymbol{e}^{\beta}$. В первом случае для координат $\epsilon_{c}^{\alpha}, e^{\beta} a(a \in J)$ справедливы равенства

$$
e^{\alpha_{a}}=e^{\beta}{ }_{a}, \quad a \in J,
$$

а во втором - отдельно нужно рассматривать случаи, когда $1 \sigma=1 \tau$ или $1 \sigma \neq 1 \tau$. Отсюда с учетом определяющих формул (4) и (8), а также условия 2) из определения 1 , получаются соответственно соотношения

$$
e^{\alpha_{1 \sigma}}=e^{\beta}{ }_{1 \sigma}, \quad e^{\alpha_{2 \sigma}}>e^{\beta_{2 \sigma}}, \quad e^{\alpha_{3 \sigma}}<e^{\beta} \beta_{3 \sigma},
$$

или

$$
\begin{aligned}
& e^{\alpha_{1 \sigma}}>e^{\beta}{ }_{1 \sigma}, \quad e^{\alpha_{1 \tau}}<e^{\beta}{ }_{1 \tau}, \\
& e^{\alpha_{a}}=r-e^{\alpha_{1 \sigma}}-e^{\alpha_{1 \tau}}, \quad e^{\beta}{ }_{a}=r-e^{\beta_{1 \sigma}}-e^{\beta}{ }_{1 \tau}, \quad a=J \backslash\{1 \sigma, 1 \tau\} .
\end{aligned}
$$

Здесь следует иметь в виду, что по (8)

$$
e^{\alpha}{ }_{a \sigma}={ }^{*} x_{a \sigma}^{\sigma}, \quad \sigma \in E^{\alpha}(\Pi), \quad a \in J,
$$

и любой полиматроид $\Pi \in \Pi(3)$ задается системой (16). Тем самым

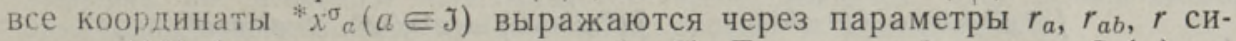
стемы (16) (они представлены в табл. 2). Если $\sigma \in \mathcal{S}_{3}$ и $\tau \in \mathcal{S}_{3}(\sigma)$, то соотношения $\boldsymbol{e}^{\alpha}=\boldsymbol{e}^{\beta}$ или $\boldsymbol{e}^{\alpha} \neq \boldsymbol{e}^{\beta}$ являются существенными в том смысле, что они с помощью (18), (19) и (20) налагают определенные условия на выражения из табл. 2. В конечном счете последние и будут искомыми нами условиями для параметров $r_{a}, r_{a b}$ и $r$ системы (16), задающей полиматроид П из гласса $L\left(m ; p^{\alpha} ; q^{\alpha}\right)$. Если $\sigma \in \mathcal{S}_{3}$ и $\tau \notin \mathcal{S}_{3}(\sigma)$, то равенство $\boldsymbol{e}^{\alpha}=\boldsymbol{e}^{\beta}$ возможно лишь при условиях теоремы 8 , и так же, как соответствующее неравенство $e^{\alpha} \neq e^{\beta}$, дополнительных условий на параметры $r_{a}, r_{a b}, r$ не налагает. Если полученные условия на параметры совместны, то класс $L\left(m ; p^{\alpha} ; q^{\alpha}\right)$ непустой, в противном случае - пустой и тогда $\left(m ; p^{\alpha} ; q^{\alpha}(\alpha \in \mathcal{M})\right)$ не служит набором параметров для структурной классификации трехмерных полиматроидов.

Наоборот, если известна система (16), задающая полиматроид $\Pi \in \Pi(3)$, то, используя табл. 3 , легко указать набор параметров $\left(m ; p^{\alpha} ; q^{\alpha}(\alpha \in \mathcal{M})\right)$ структурного класса, в который входит рассматриваемый полиматроид, так как в таблице перечислены все возможные разбиения $S_{3}$ на классы эквивалентности $E^{\alpha}(\Pi)$.

Следует заметить, что в общем не нужно отдельно рассматривать все 18 наборов. Это число сокращается, если сперва ограничиться описанием классов эквивалентности $E^{\alpha}(\Pi)$ только с помощью параметров $\left(m ; p^{\alpha}(\alpha \in \mathcal{M})\right)$ (см. $\left.\left[{ }^{9}\right]\right)$. Если по вышеописанной схеме получается противоречивый набор условий на $r_{a}, r_{a b}, r$, то и соответствующие классы $L\left(m ; p^{\alpha} ; q^{\alpha}\right)$ будут пустыми. Таким образом, из рассмотрения исключаются наборы $2,3,5,6,7$ и 12 из предложения 10, так как наборы 
$\left(m ; p^{\alpha}(\alpha \in \mathcal{M})\right)$ с $(2 ; 4,2),(3 ; 4,1,1),(3 ; 3,2,1)$ и $(4 ; 3,1,1,1)$ приводят к противоречивым условиям для $r_{a}, r_{a b}$ и $r$. Наборами структурных классов не будут также служить наборы 9 и 10 из списка предложения 10 , так как с учетом теорем 7 и 8 невозможно построить множества классов эквивалентности $E^{\alpha}(\Pi) \subset \mathcal{S}_{3}(\alpha \in \mathcal{M})$ с соответствующими характеристиками. Как уже отмечалось, из наборов предложения 10 только 10 служат наборами параметров структурной классификации трехмерных полиматроидов. Для всех их можно доказать предложение, аналогичное следующему.

Предложение 11. Многогранник П содержится в классе $L(5$; $2,1,1,1,1 ; 1,1,1,1,1) \subset \Pi(3)$ тогда и только тогда, когда он задается такой системой (16), в которой существует $k \in J$ так, что

$$
\begin{array}{cl}
r_{i}+r_{j}>r_{i j}, \quad r_{i}+r_{k}>r_{i k}, & r_{j}+r_{k}>r_{j k}, \\
r<r_{i j}+r_{i k}-r_{i}, & r<r_{i j}+r_{j k}-r_{j}, \quad r=r_{i k}+r_{j k}-r_{k} .
\end{array}
$$

По вышесказанному, достаточность условий следует из табл. 3 , так как условия предложения соответствуют случаю $4 \mathrm{~B}$, определяющему классы $E^{\alpha}(\Pi) \subset \mathcal{S}_{3}$ с указанными характеристиками. Чтобы доказать необходимость, обозначим

$$
\begin{aligned}
& E^{1}(\Pi)=\{(k, i, j),(k, j, i)\}, \quad E^{2}(\Pi)=\{(i, k, j)\}, \\
& E^{3}(\Pi)=\{(i, j, k)\}, \quad E^{4}(\Pi)=\{(j, i, k)\}, \quad E^{5}(\Pi)=\{(j, k, i)\} .
\end{aligned}
$$

Тогда действительно $m=5, p^{1}=2, p^{2}=\ldots=p^{5}=1, q^{1}=\ldots$ $\ldots=q^{2}=1$ и по (8)

$$
\begin{aligned}
& e^{1}=f\left({ }^{*} x^{(k, i, j)}\right)=f\left({ }^{*} x^{(k, j, i)}\right), \quad e^{2}=f\left({ }^{*} x^{(i, k, j)}\right), \\
& e^{3}=f\left({ }^{*} x^{(i, j, k)}\right), \quad e^{4}=f\left({ }^{*} x^{(j, i, k)}\right), \quad e^{5}=f\left({ }^{*} x^{(j, k, i)}\right) .
\end{aligned}
$$

При этом существенными соотношениями будут

$$
\begin{gathered}
f\left({ }^{*} x^{(k, i, j)}\right)=f\left({ }^{*} x^{(k j, i,)}\right), \quad f\left({ }^{*} x^{(k, i, j)}\right)=e^{1} \neq e^{2}, \quad e^{2} \neq e^{3}, \\
e^{3} \neq e^{4}, \quad e^{4} \neq e^{5}, \quad e^{5} \neq e^{1}=f\left({ }^{*} x^{(k, j, i)}\right) .
\end{gathered}
$$

\begin{tabular}{|c|c|c|c|c|c|}
\hline 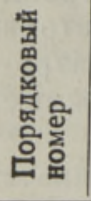 & $\begin{array}{c}\text { Параметры класса } \\
\qquad\left(m ; p \alpha ; q^{\alpha}\right)\end{array}$ & 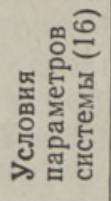 & 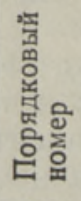 & $\begin{array}{c}\text { Параметры класса } \\
L\left(m ; p \alpha ; q^{\alpha}\right)\end{array}$ & 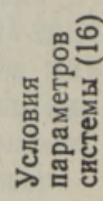 \\
\hline $\begin{array}{l}1 . \\
2 . \\
3 . \\
4 . \\
5 .\end{array}$ & $\begin{array}{l}(1 ; 6 ; 3) \\
(2 ; 3,3 ; 2,2) \\
(3 ; 2,2,2 ; 2,2,2) \\
(3 ; 2,2,2 ; 1,1,1) \\
(4 ; 2,2,1,1 ; 2,2,1,1)\end{array}$ & $\begin{array}{l}1 \mathrm{~A} \\
2 \mathrm{~A} \\
1 \mathrm{~B} \\
4 \mathrm{~A} \\
2 \mathrm{~B}\end{array}$ & $\begin{array}{l}6 . \\
7 . \\
8 . \\
9 . \\
10 .\end{array}$ & $\begin{array}{l}(4 ; 2,2,1,1 ; 2,1,1,1) \\
(4 ; 2,2,1,1 ; 1,1,1,1) \\
(5 ; 2,1,1,1,1 ; 2,1,1,1,1) \\
(5 ; 2,1,1,1,1 ; 1,1,1,1,1) \\
(6 ; 1,1,1,1,1,1 ; 1,1,1,1,1,1)\end{array}$ & $\begin{array}{l}3 \mathrm{~A} \\
4 \mathrm{~B} \\
3 \mathrm{~B} \\
4 \mathrm{~B} \\
4 \Gamma\end{array}$ \\
\hline
\end{tabular}

С учетом табл. 2, формул (21) и (18), (19), (20) они приводят к условиям предложения, что и требовалось.

Представим окончательный результат в табл. 4, указывая условия, при которых система (16) задает полиматроид из соответствующего класса с помощью номера из табл. 3 (см. $\left.\left[{ }^{10}\right]\right)$.

Tаблица 4

Полный список структурных классов трехмерных полиматроидов 


\section{ЛИТЕ РА Т У А}

1. Емеличев В. А., Ковалев М. М., Кравцов М. К. Многогранннки, графы, оптимизация. М., «Наука», 1981.

2. Емеличев В. А., Ковалев М. М. Кнбернетика, № 6, 54-62 (1982).

3. Dathe, H., Girlich, E. In: 27. Intern. Wiss. Koll. TH Ilmenau. Vortragsreihe «Mathematische Optimierung-Theorie und Anwendung». Ilmenau, 1982, 137139.

4. Hoppe, V., Kowaljow, M. M., Girlich, E. Friedrich-Schiller-Univ. Jena. Forschungsergebnisse, № N/81/40, 1-18 (1981).

5. Ковалев М. М., Гирлих Э. Изв. АН БССР. Сер. физ.-мат. н., № 4, 5-10 (1980).

6. Dathe, H.. Girlich, E., Kowaljow, M. M. Wiss. Z. Friedrich-Schiller-Univ. Jena. Math-Naturwiss. Reihe, № 4, 657-666 (1982).

7. Gurlich, E. Friedrich-Schiller-Univ. Jena. Forschungsergebnisse, № 59 (N) 78, $1-11(1978)$

8. Girlich, E., Kowaljow, M. M. Math. Operationsforsch. und Statistik. Ser. Optim:zation, 11, № 4. 547-561 (1980).

9. Рийвес K. В кн.: 350 лет математики в Тартуском университете. Тез. докл. Тарту, Изд. ТГУ, 1982, 46-49.

10. Riives, $K$. In: 27. Intern. Wiss. Koll. TH Ilmenau. Vortragsreihe «Mathematische Optimierung-Theorie und Anwendung». Ilmeau, 1982, 23-26.

11. Welsh, D. J. A. Theory of Matroids. London-New York-San Francisco, Acad. Press, 1976.

\section{Эстонская сельскохозяйственная академия}

\section{Поступила в редакцию $8 / \mathrm{VI} 1983$}

\section{Kaarin RIIVES}

\section{POLUMATROIDIDE KLASSIFITSEERIMISULESANNE}

Polümatroidid ["1] on üheselt määratud oma sōltumatutest elementidest moodustatud kumerate hulktahukate poolt. Seepärast on polümatroidide klassifitseerimisülesanne samaväärne sõltumatute elementide hulktahukate klassifitseerimisülesandega. Olesande lahendamiseks on kasutatud arvuliste karakteristikute süsteeme, mis täielikult kirjeldavad vaadeldavate hulktahukate kombinatoorset struktuuri. Spetsiaalselt valitud karakteristikute huigad määravad mitmeid omavahel seotud klassifikatsioone. On kirjeldatud ühe vōimaliku, nn. struktuurse klassifikatsiooni saamise pōhietappe ning näitena esitatud üksikasjaiik kolmemõōtmeliste polümatroidide struktuurne klassifikatsioon.

\section{Kaarin RIIVES}

\section{A CLASSIFICATION PROBLEM OF POLYMATROIDS}

Polymatroids [ $\left.{ }^{11}\right]$ are uniquely determined by their convex polytopes of independent elements. So the classification problem of polymatroids is equivalent to the classification problem of these polytopes. In the paper this problem is solved with the help of special characteristics systems which describe completely the combinatorial structure of the polytopes. Specially fixed sets of the characteristics determine some classifications closely connected with one another. In the paper there are described the basic stages of receiving one of these classifications which is termed to be a structural classification. As an example, the structural classification of 3 -dimensional poiymatroids is given. 